\title{
RELATIVISTIC ELECTRONS OF THE OUTER RADIATION BELT AND METHODS OF THEIR FORECAST (REVIEW)
}

\author{
A.S. Potapov \\ Institute of Solar-Terrestrial Physics SB RAS, Irkutsk, Russia, \\ potapov@iszf.irk.ru
}

The paper reviews studies of the dynamics of relativistic electrons in the geosynchronous region. It lists the physical processes that lead to the acceleration of electrons filling the outer radiation belt. As one of the space weather factors, high-energy electron fluxes pose a serious threat to the operation of satellite equipment in one of the most populated orbital regions. Necessity is emphasized for efforts to develop methods for forecasting the situation in this part of the magnetosphere, possible predictors are listed, and their classification is given. An example of a predictive model for forecasting relativistic electron flux with a 1-2-day lead time is proposed. Some questions of practical organization of prediction are discussed; the main objectives of short-term, medium-term, and long-term forecasts are listed.

Keywords: Radiation belts $\cdot$ Relativistic electrons $\cdot$ Forecast $\cdot$ Magnetosphere $\cdot$ Solar wind

\section{INTRODUCTION}

One of the first scientific achievements at the onset of the space age was the discovery of terrestrial radiation belts made by J. Van Allen and teams under the charge of S.N. Vernov and V.I. Krasovsky in 1958 [Temny, 1993]. V.I. Krasovsky was the first to suggest that electrons detected by the third Soviet satellite could not reach the dense atmosphere as they had been captured by the geomagnetic field. This discovery led to the formation of a full-fledged field of research into the dynamics of energetic particle fluxes in the geomagnetic field. On the one hand, the permanent presence of charged particle fluxes in the magnetosphere is the most important characteristic of its state, and on the other, radiation belts can pose a serious threat to moon-bound manned spacecraft and hinder normal operation of satellite equipment.

Earth's magnetosphere is thought to have two constantly existing radiation belts: the inner belt formed mainly by protons, and the outer one filled by energetic electrons. This division is largely arbitrary since, for example, the magnetic shell $L=1.2-1.5$ exhibits a stable electron belt with $E>20 \mathrm{MeV}$ [Galper et al., 1983]. The position of the proton flux maximum depends on their energy and shifts from $L=1.5$ to $L=3$ with energy decreasing from $100 \mathrm{MeV}$ to $1 \mathrm{MeV}$. The peak intensity of electron fluxes for almost the entire energy range (from $40 \mathrm{keV}$ to $2 \mathrm{MeV}$ ) is within magnetic shells $L=5-6$. At geosynchronous orbit, the $\sim 1 \mathrm{MeV}$ electron density is by three orders of magnitude higher than the proton density with the same energy. This maintains roughly equal energy density of these particles [Borovsky et al., 2016]. As inferred from observations of geosynchronous satellites, the electron energy spectrum in the range from $50 \mathrm{keV}$ to $1.5 \mathrm{MeV}$ obeys a power law; the exponent can vary widely from -6 to -2 [Freeman et al., 1998; Xiao et al., 2008]. 


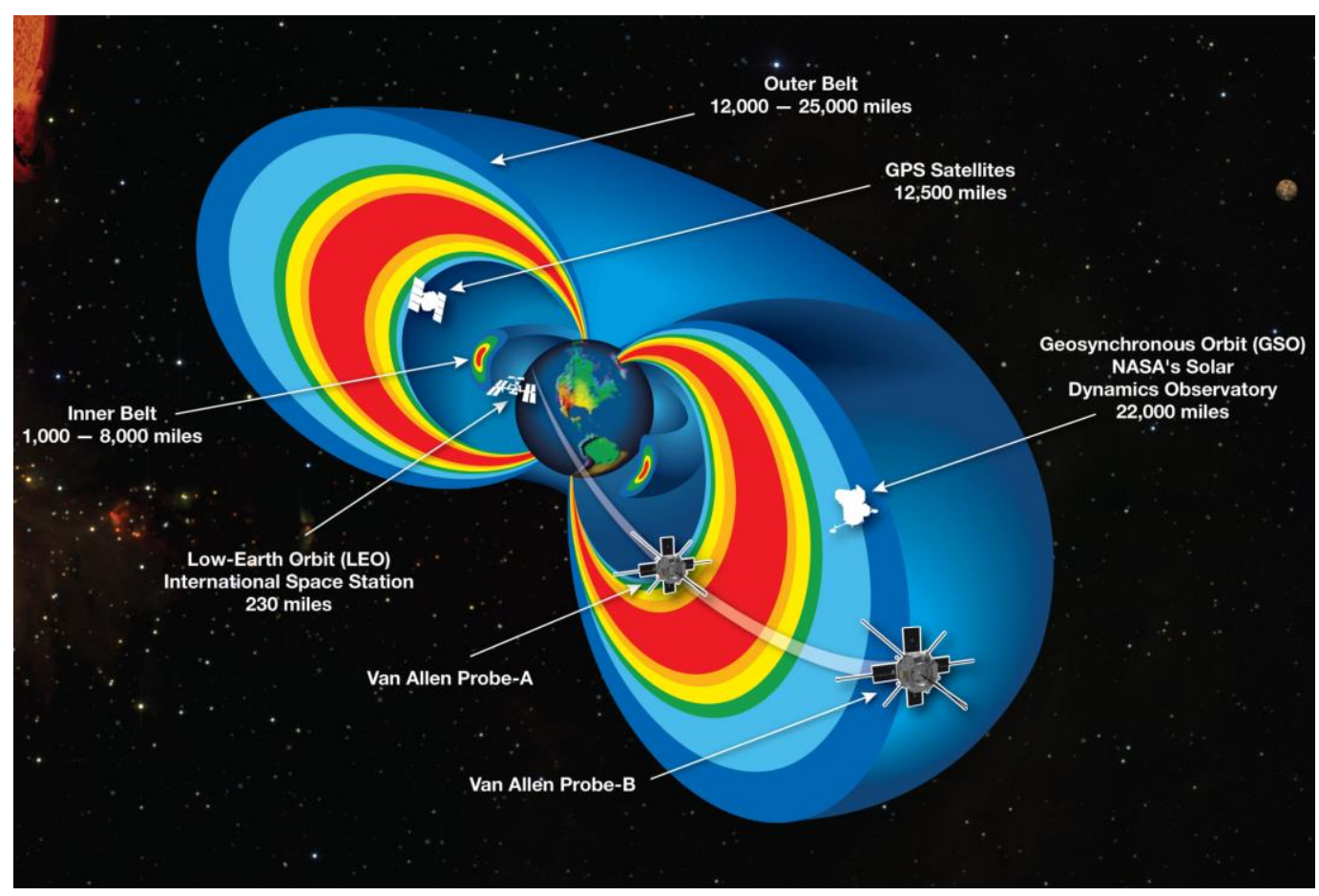

Figure 1. Location of radiation belts and orbits of different spacecraft [https://encryptedtbn0.gstatic.com/images?q=tbn:ANd9GcQRReeeIY4NuZm-hTm8qUT6aNUkZ53jtpeCd8eo6d-pL88X2DVNZw]

In this review, we restrict ourselves to the analysis of the role of radiation belts in the formation of space weather. High-energy electrons in the outer radiation belt are often called killer electrons. Why do relativistic electrons represent a threat? Forty percent of all near-Earth satellites are at geosynchronous orbit, i.e. in the outer part of the outer radiation belt.

They perform communication, navigation, reconnaissance, and other functions. Relativistic electrons can cause deep dielectric charging of satellites and damage them or lead to malfunction. Furthermore, energetic charged particles induce degradation of spacecraft solar batteries and generate impulse noise in data acquisition and transmission systems. Figure 1 shows the location of the radiation belts and orbits of different spacecraft.

All this necessitates predicting the behavior of relativistic electrons at geosynchronous orbit. One of the first who drew attention to the hazards inherent in the outer radiation belt enhancement was D.N. Baker [Baker et al., 1987; Baker, 2000, 2001; Baker et al., 1998, 2001]. In our country, a large series of research works on the effect of energetic particle fluxes on spacecraft operation was carried out at the Institute of Physics of the Earth of the Russian Academy of Sciences by V.A. Pilipenko and N.V. Romanova [Pilipenko, Romanova, 2005; Romanova et al., 2005; Pilipenko et al, 2006.; Romanova, Pilipenko, 2009; Romanova et al., 2009]. In recent years, many papers have been published on different methods of forecasting sudden enhancements of charged particle fluxes in the radiation belts. First of all, it was found that the energetic-electron population of the outer radiation belt increases with Earth's magnetosphere immersed in high-speed solar wind streams, which cause geomagnetic storms [Paulikas, Blake, 1979; Mathie, Mann, 2001; Miyoshi, Kataoka, 2005, 2008; Reeves et al., 2013]. However, only half of magnetic storms leads to an enhancement of the outer radiation belt [Reeves et al., 2003]. The rest either retain the intensity of the belt at the prestorm level or even bring about weakening of electron fluxes. Therefore, along with the processes 
triggered by magnetic disturbances, the refilling of the radiation belts is driven by other processes. The outstanding complexity of the interaction between the solar wind and the magnetosphere, which causes acceleration of charged particles and refiling of the radiation belts with them, produces multifactorial dependence of relativistic fluxes on the state of the magnetosphere and interplanetary medium. At the same time, this dependence is ambiguous. This even more significantly impedes the development of methods for radiation belt forecasting.

This review describes current knowledge of only one aspect of the problem of radiation belts, namely of the dynamics of the high-energy electron component in the outer radiation belt, which is revealed by measurements at geosynchronous orbit, as well as methods for forecasting relativistic electron fluxes proposed to date. First, we review the main characteristics of the outer radiation belt, and then discuss its dynamics during geomagnetic disturbances. Next, we describe the existing approaches to electron flux forecasting, discuss the choice of predictors for this forecast and, finally, propose an example of the predictive model enabling us to estimate relativistic electron fluxes with a 1-2-day lead time.

\section{MAIN CHARACTERISTICS OF THE OUTER RADIATION BELT}

\subsection{Energy sources and sinks}

The state of the outer radiation belt, i.e. flux intensity, density of electron component particles, is conditioned by the dynamic equilibrium between particle sources and electron losses due to various processes. Both are extremely diverse, thus further hindering the forecasting of charged particle fluxes. Particle sources can be classified into two groups: primary sources ensuring the arrival of low-energy electrons (under $10 \mathrm{keV}$ ), and secondary ones represented by processes of electron acceleration up to relativistic energies (over $1 \mathrm{MeV}$ ). The primary sources involve capturing particles from the interplanetary medium when the magnetopause shifts during sudden compressions of the magnetosphere by the solar wind [Tverskoi, 1964], injecting particles from the geomagnetic tail to the region of trapped radiation [Tverskoi, 1968], and transporting charged particles in the magnetosphere with diffusion under the influence of nonstationary electric fields [Parker, 1960; Schulz, Lanzerotti, 1974].

Virtually all the secondary sources described in literature are associated with stochastic radial transport of drifting electrons to magnetic shells, which are closer to Earth, due to wave-particle interaction, but researchers propose a wide variety of mechanisms for this interaction [Schulz, Lanzerotti, 1974 Brautigam, Albert, 2000; Ozeke et al., 2014]. The recirculation model is often mentioned [Fujimoto, Nishida, 1990]: slow electron diffusion across magnetic shells at low heights under the action of electric field fluctuations with a quasi-period of $1 \mathrm{~s}$ and an intensity of a few tens of millivolts per meter, which is comparable with the amplitude of geomagnetic pulsations at high latitudes. This model was repeatedly supplemented with other mechanisms, for example, Ukhorskiy et al. [2014] suggest taking into account bifurcations of drift orbits in the model. This increases by an order of magnitude the radial drift velocity and energy accumulation for large values of the pitch angle of particles in the equatorial region. Acceleration drivers, i.e. those waves with which accelerating electrons interact, can be both long-period Pc4-Pc5 ULF pulsations [Elkington et al., 1999; Hudson et al., 2000; Mathie, Mann, 2001; Gubar, 2010], and VLF waves [Horne, Thorne, 1998; Summers et al., 2007; Simms et al., 2015]. ULF oscillations accelerate electrons through the driftresonance mechanism when the period of particle drift around Earth coincides with the period of ULF oscillations [Elkington et al., 1999]. The period of rotation of relativistic charged particles around Earth (in minutes) is [Kuznetsov, 2010] 


$$
\tau=\frac{88\left(1+E / E_{0}\right)}{2+E / E_{0}} \frac{K}{L E},
$$

where $K=1.25-0.25 \cos ^{2} \lambda_{\mathrm{m}}$. Here $E$ is the particle energy $(\mathrm{MeV}), E_{0}$ for electron is $0.51 \mathrm{MeV}, \lambda_{\mathrm{m}}$ is the geomagnetic latitude of reflection point. For electrons with $E \sim 2 \mathrm{MeV}$ the period of rotation $\tau$ is $\sim 5-7$ minutes, this is a range of Pc5 geomagnetic pulsations.

In turn, whistler modes of VLF radiation can enter into resonance with the gyrofrequency of electrons and accelerate them through electron cyclotron instability [Li et al., 2005]. Many authors believe that electrons are simultaneously accelerated to relativistic velocities due to the double effect of ULF and VLF oscillations [O'Brien et al., 2003; Li et al., 2005; Roeder et al., 2005].

However, the same oscillations, depending on specific conditions, can also weaken the energetic electron flux in the radiation belt, taking the particle energy or converting electrons into the loss cone, i.e. they can act as one of the channels of radiation belt energy sink [Roeder et al., 2005]. There is a recorded case [Shprits et al., 2016] when ion-cyclotron waves led to an increase in the population of relativistic electrons with energies of $\sim 1 \mathrm{MeV}$ and at the same time induced scattering of ultra-relativistic electrons with an energy over $4 \mathrm{MeV}$ into the loss cone. Other causes of the decrease in the relativistic electron flux at geosynchronous orbit are: 1) particle losses at the magnetopause and 2) "swelling" of electron drift orbits under the impact of the increased ring current field [Ukhorskiy et al., 2015]. Both the processes usually occur during the main phase of a geomagnetic storm.

Let us also mention the exotic models that invoke Jovian relativistic electrons [Baker et al., 1979] and solar wind electrons penetrating through cusp [Sheldon et al., 1998] to explain the dynamics of the terrestrial outer radiation belt.

\subsection{Behavior of the radiation belt during disturbances}

Magnetic storms significantly change electron belt conditions, depopulating or populating it [Frieda et al., 2002; Reeves et al., 2003]. However, only half of the storms lead to the final relativistic electron flux enhancement at geosynchronous orbit. Figure 2 shows the typical behavior of energetic electron fluxes $J_{\text {ell }}$ during a magnetospheric storm, which was obtained by the epoch superposition method for 31 magnetic storms. All these storms were generated by magnetospheric effects of corotating interaction regions (CIR) of fast and slow solar wind [Potapov, 2013]. For the 0-epoch we take the moment of contact between the front of CIR solar wind stream and the magnetopause.

The sudden drop in the electron flux at the initial phase of the storm is attributed to the above-mentioned effect of the ring current field, which increases sharply during the storm, particle losses at the magnetopause playing the main role at geosynchronous orbit [Ukhorskiy et al., 2015]. Electrons during their azimuthal drift are brought out of the last closed drift shell and cross the magnetopause having approached Earth, going into the interplanetary medium [Turner et al., 2012; Kessel, 2016]. These losses are aggravated by outward radial diffusion, which pushes the particles to this loss zone through the magnetopause [Schulz, Lanzerotti, 1974 Kessel, 2016]. 

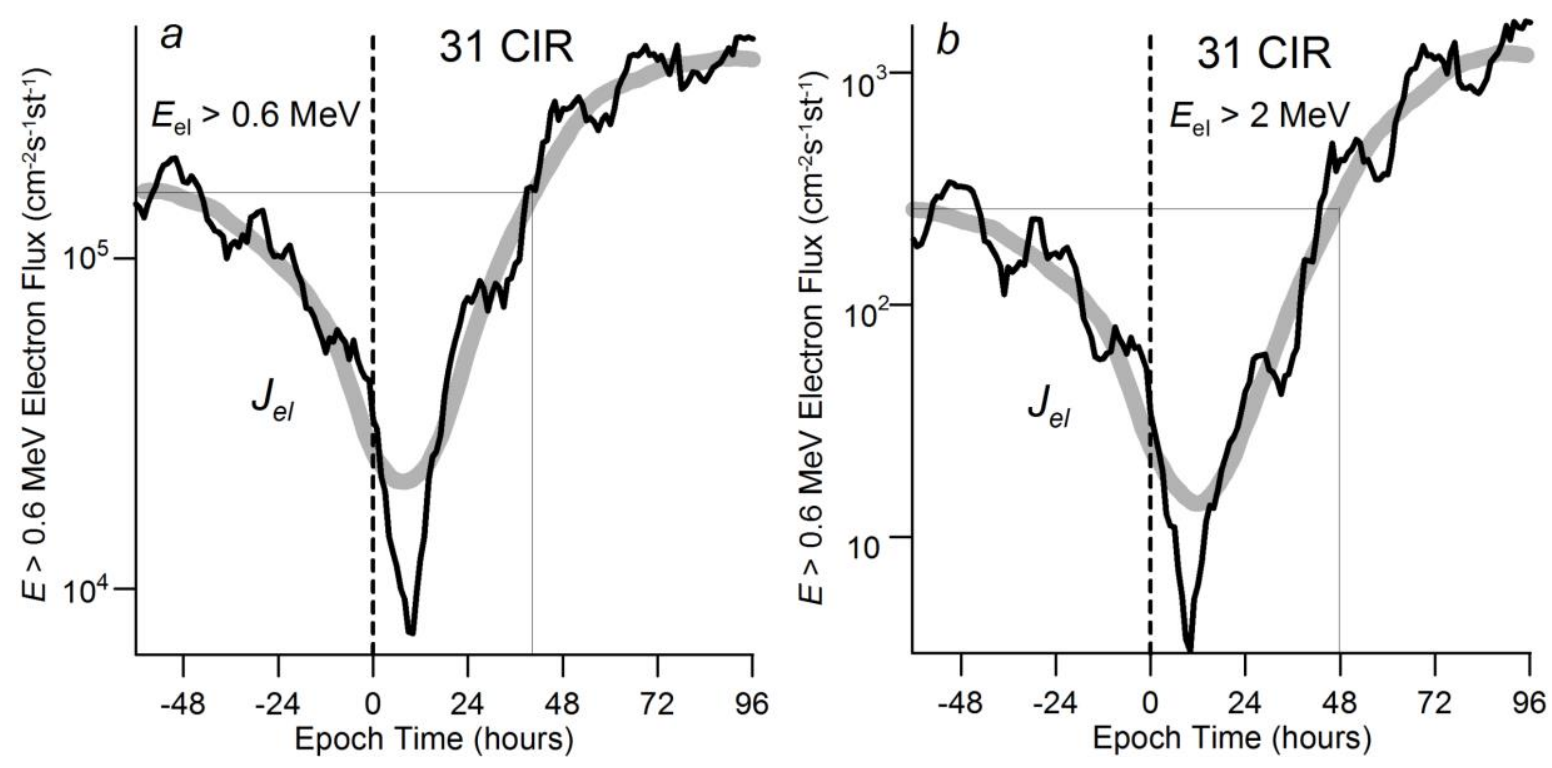

Figure 2. Variations of magnetospheric electron fluxes with energies over $600 \mathrm{keV}(a)$ and $2 \mathrm{MeV}(b)$ during the period of CIR high-speed solar wind streams, obtained by the method of superposed epochs from measurements at geostationary satellites GOES-8 and -12: black curves indicate hourly average values, gray ones show the moving average over 25 points [Potapov, 2013]

The sharp drop in the electron fluxes during the main storm phase is followed by their enhancement. It concurs with the recovery phase of the magnetic storm. Electron fluxes with moderate energies recover somewhat faster, on average for 40 hours as compared to two days in the case of particles with energy higher than $2 \mathrm{MeV}$. As noted above, a key role in filling the external radiation belt belongs to two processes: 1) drift resonance interaction of so-called seed electrons (particles with energies from tens to hundreds of electron volts) with Pc5 pulsations [Elkington et al., 1999; Hudson et al., 2000; Gubar, 2010]; 2) cyclotron resonance of electrons with VLF emissions - whistler and chorus waves, and other VLF types, as well as with ion-cyclotron waves [Horne, Thorne, 1998; Summers, Ma, 2000; Summers et al., 2007].

We cannot also rule out the contribution of stochastic mechanisms of acceleration due to electron flux effects of pulses or irregular field perturbations, which lead to breakdown of the third invariant, excursion of orbits of trapped particles to lower heights with simultaneous acceleration [Green, Kivelson, 2004]. In any case, all the mechanisms of electron acceleration to relativistic velocities require the presence of seed electrons in the magnetosphere, which are usually represented by particles injected from the geomagnetic tail during substorms.

\section{PROBLEM OF PREDICTING HIGH-ENERGY ELECTRON COMPONENT OF THE OUTER RADIATION BELT}

To date, literature has offered dozens of methods for forecasting electron fluxes in the outer radiation belt [Baker et al., 1990; Ling et al., 2010; Weigel et al., 2003; Ukhorskiy et al., 2004; Li et al., 2001; Perry et al., 2010; Sakaguchi et al., 2015; Efitorov et al., 2016; Potapov et al., 2016]. They differ in: 1) selection of predictors; 2) forecasting methods; 3) lead time. The number of predictors in use varies from one [http: //www.swpc.noaa.gov/products/Relativistic-Electron-forecast-the model] to 26 [Simms et al., 2014]. As forecasting 
methods researchers most often employ linear filters [Baker et al., 1990], formed on the basis of the multivariate analysis [Simms et al., 2014, 2016; Potapov et al., 2016], in particular using the Kalman filter [Sakaguchi et al., 2015], as well as nonlinear methods such as neural networks [Ling et al., 2010; Wide et al., 2016] and nonlinear autoregressive moving average modeling (NARMAX) [Balikhin et al, 2011]. By the lead time, forecasts are divided into short-, medium-, and long-term.

\subsection{Predictors of relativistic electron flux enhancements}

Selection of predictors is an important preliminary procedure of any forecast. Which predictors are commonly used for devising methods of forecasting relativistic electron fluxes?

It is clear that electron acceleration processes begin working only under non-stationary conditions. Magnetospheric disturbances can contribute to the increase in population of the outer radiation belt in two ways. First, during magnetic storms and substorms, seed electrons from the tail are ejected and captured in the geomagnetic field. This is followed by precipitation of particles in auroral regions with simultaneous generation of transient ionospheric currents and irregular magnetic variations over a wide frequency range. Hence, indicators of upcoming rise in the energetic electron density at geosynchronous orbit can be auroral activity indices such as $A E$ [Baker, et al., 1990; Li et al., 2009], as well as indices of activity of irregular Pi1 geomagnetic pulsations [Degtyarev et al., 2009a, b, 2010]. Second, during disturbances, the intensity of various pulses and nonstationary magnetic field oscillations grows significantly. This can trigger stochastic mechanisms of electron acceleration. The level of such disturbances is best monitored by such indices as the planetary index $K_{\mathrm{p}}$ and the ring current intensity index $D s t$; therefore these indices can also be used as predictors [Nagai, 1988; Lyatsky, Khazanov, 2008].

However, as already noted, only half of magnetic storms lead to an enhancement of energetic electron flux as compared to the prestorm period, and, moreover, there is no direct correlation between storm intensity and degree of flux enhancement. Accordingly, there should be some additional factors capable of acting as predictors.

From a physics perspective, the best predictors are indices of those processes which are involved in the acceleration of electrons to relativistic energies and can explain variations in particle fluxes at geosynchronous orbit. They are primarily the amplitude of Pc4-5 ULF oscillations (a long-term series of indices characterizing the intensity of these oscillations on Earth, in the solar wind, and at geostationary orbit, is given on the website [ulf.gcras.ru]), the amplitude of VLF emissions (whistler and chorus waves, etc.), and indices of magnetic disturbance. All of them in certain models can be recalculated into effective electron diffusion coefficients, i.e. they can characterize the diffusion pumping of particle energy. From a statistical point of view, the best predictors are the parameters which have the highest correlation with relativistic electron fluxes alone or in conjunction with other parameters in the multidimensional correlation analysis. In reality, the two criteria for optimal selection of predictors do not always coincide. So, based on pair correlation coefficients, the solar wind velocity $V_{\mathrm{sw}}$ has the closest relationship with the electron flux [Paulikas, Blake, 1979; Lyons et al., 2005; Reeves et al., 2011, 2013; Kellerman, Shprits, 2012]. However, there is no reasonable mechanism capable of explaining the direct effect of the velocity at which the solar wind plasma flows round the magnetosphere on the intensity of the outer radiation belt. There is surely an indirect effect. It is primarily related to the fact that high-speed solar wind streams bring ultra-low frequency MHD waves, which 
either partially penetrate into the magnetosphere or modulate ULF activity within the magnetosphere [Potapov et al., 2012; Potapov, 2013]. The intensity of ULF oscillations both in the solar wind and on Earth is just the second parameter with respect to the strength of the relationship with the density of relativistic electrons [Mann et al., 2004; Romanova et al., 2007; Romanova, Pilipenko, 2009; Potapov et al., 2012].

Thus, predictors can be direct, describing the direct effect on a predicted process, and proxies characterizing some of features of the process and replacing the direct predictors if information about the latter is not available or sufficient. In our case, as the proxy-predictor we can take the solar wind speed, despite its having the highest correlation coefficient with the electron flux, while amplitudes of ULF oscillations and VLF radiation are direct predictors. One set of predictive parameters often includes predictors of both types.

Another important feature of predictive parameters is how far in advance they can warn us of a change of the parameter predicted (in our case, the relativistic electron flux), i.e. the forecast lead time $\Delta t$. So, for example, for the amplitude of ULF oscillations on Earth and in the solar wind, the lead time is 2-3 days, for the solar wind velocity $\Delta t=2$ days, and the lead time $\Delta t$ of changes of the ecliptic component of the interplanetary magnetic field (IMF) is 0 days, i.e. this parameter varies simultaneously with the electron flux in the magnetosphere.

The table lists the predictors most commonly used for working out methods of forecasting the relativistic electron flux. It also identifies their type (direct or proxy) and the approximate value of the forecast lead time. Question marks indicate either the absence of data or discrepancy between sources.

\subsection{Forecast lead time}

As noted above, forecasts can be short-, medium-, and long-term.

The short-term forecast is needed for rapid response. After receiving advance alarm, operators of satellite systems can prepare themselves for disruptions, put the onboard equipment into low power mode, and activate safety equipment. Some predictors have a two-three-day lead time with respect to enhancement of relativistic electron fluxes; therefore we can produce a forecast with a lead time of up to three days. It can be promptly corrected if first it is made using only three-day advance predictors and then is adjusted involving 48- and 24-hour predictors. Next, we propose an example of how a short-term forecast is prepared.

The medium-term forecast in our case gives an alarm about possible approach of a high-speed solar wind stream to the magnetosphere. The lead time of this forecast can be up to 5-6 days if current data on the direction of propagation of coronal mass ejection or the stream path from the coronal hole are available. This forecast relies only on observations of coronal mass ejections and coronal holes. Its technique actually coincides with the forecast of geomagnetic disturbances that is based on the monitoring of the Sun's behavior. At the moment when the front of a high-speed solar wind stream interacts with the magnetosphere, the short-term forecast process begins.

The long-term forecast is not so much a prediction as recommendations for planning space projects. These recommendations can arise from the data on the dependence of relativistic electron flux frequency and intensity on the solar cycle phase, in combination with the forecast for the cycle itself. To date, we have enough measurements of particle fluxes to get an idea about their cyclic variations. Figure 3 gives an example of how the average monthly flux of relativistic electrons varied during solar cycle 23. 
Data on long-term variations of correlation coefficients between electron flux and different predictors may also be useful for the long-term forecast [Potapov et al., 2014; Myagkova, Dolenko, 2016].

Predictors often used for predicting the relativistic electron flux at geosynchronous orbit

\begin{tabular}{|c|c|c|c|c|c|}
\hline Solar wind (SW) parameters & $\begin{array}{l}\text { Lead } \\
\text { time, } \\
\text { days }\end{array}$ & $\begin{array}{l}\text { Type of } \\
\text { predictor }\end{array}$ & Magnetospheric parameters & $\begin{array}{l}\text { Lead } \\
\text { time, } \\
\text { days }\end{array}$ & $\begin{array}{l}\text { Type of } \\
\text { predictor }\end{array}$ \\
\hline SW speed $V_{\text {sw }}$ & 2 & Proxy & Auroral electrojet index $A E$ & $?$ & Proxy \\
\hline SW plasma density $N_{\mathrm{p}}$ & 1 & Proxy & Planetary index $K_{\mathrm{p}}$ & $?$ & Proxy \\
\hline $\begin{array}{l}\text { IMF southward component } \\
B_{\mathrm{z}}\end{array}$ & 1 & $\begin{array}{l}\text { Proxy } \\
(?)\end{array}$ & Geomagnetic index $D s t$ & $?$ & Proxy \\
\hline $\begin{array}{l}\text { Projection of the IMF } B_{\mathrm{t}} \\
\text { vector on the plane of the } \\
\text { ecliptic }\end{array}$ & 0 & Proxy & $\begin{array}{l}\text { Maximum daily flux of seed electrons } \\
J_{\mathrm{el}}\end{array}$ & 1 & Direct \\
\hline $\mathrm{SW}$ variations $\delta V_{\mathrm{sw}} / V_{\mathrm{sw}}$ & 0 & $\begin{array}{l}\text { Proxy } \\
(?)\end{array}$ & Amplitude of ULF on the ground $A_{\mathrm{gr}}$ & 2(3) & Direct \\
\hline Variations of IMF $\delta B / B$ & 1 & Proxy & $\begin{array}{l}\text { Amplitude of ULF oscillations at the } \\
\text { geostationary orbit } A_{\mathrm{gs}}\end{array}$ & 2 & Direct \\
\hline SW dynamic pressure $P_{\text {dyn }}$ & 1 & $\begin{array}{c}\text { Direct } \\
(?)\end{array}$ & $\begin{array}{l}\text { Intensity of VLF oscillations on the } \\
\text { ground } I_{\mathrm{gr}}\end{array}$ & $?$ & Direct \\
\hline ULF amplitude in SW $A_{\text {sw }}$ & $2(3)$ & Proxy & $\begin{array}{l}\text { Intensity of VLF oscillations in the } \\
\text { magnetosphere } I_{\mathrm{ms}}\end{array}$ & $?$ & Direct \\
\hline Interplanetary electric field & 1 & Proxy & $\begin{array}{l}\text { Horizontal magnetic field component } \\
H_{\mathrm{p}} \text { at geosynchronous orbit }\end{array}$ & & Direct \\
\hline Variations of $N_{\mathrm{p}}$ & 1 & Proxy & Amplitude of Pi1 pulsations $A_{\text {Pi1 }}$ & & Proxy \\
\hline
\end{tabular}

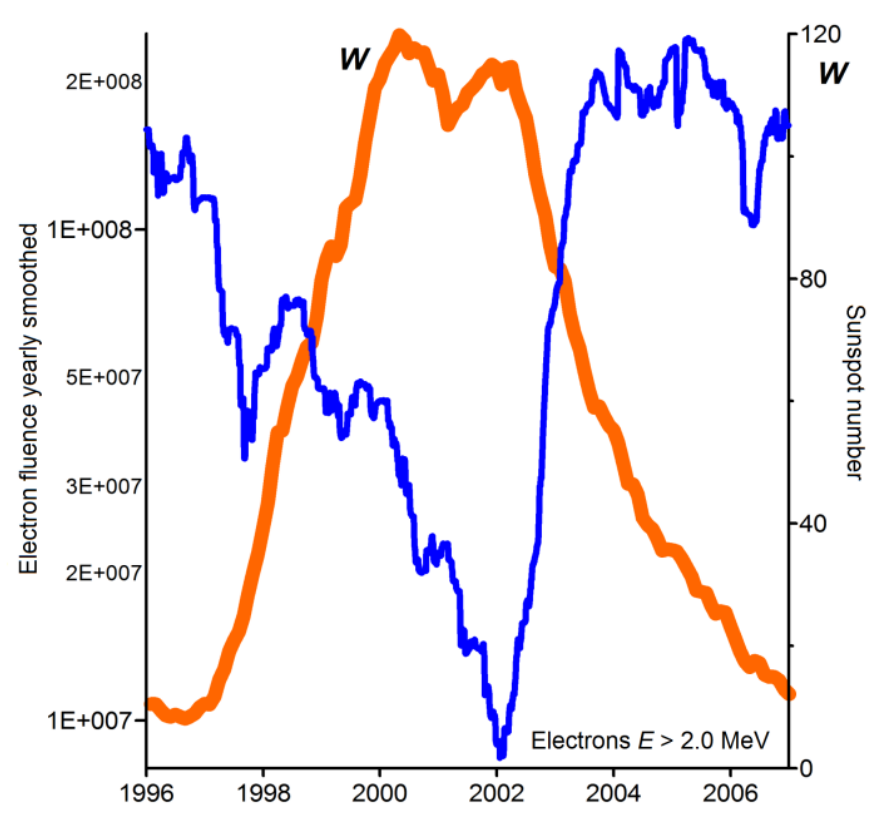

Figure 3. Solar-cyclic variation of daily integral electron flux with energy of $2 \mathrm{MeV}$ at geosynchronous orbit is indicated by the blue curve. The orange curve depicts the variation of sunspot number (Wolf number) 
Figure 4 shows how the solar wind velocity and the cumulative amplitude of on-ground ULF oscillations in the Pc5 range (150-600 s) is correlated with the relativistic electron fluxes during the solar cycle. It is seen that, first, the correlation level is approximately equal for the solar wind velocity and ULF amplitude, and, second, the correlation is minimum during maximum solar activity and maximum during minimum solar activity and during the decay phase of solar cycle 23 . The cumulative amplitude is calculated as

$$
A_{\text {int }}=\sum_{j=i-3 \mu}^{i} \log [A(i)] \exp \left(-\frac{i-j}{\mu}\right)
$$

where $\mu$ is the period of time over which data on amplitude are accumulated to obtain $A_{\text {int }}$ [Romanova et al, 2007.]. The authors of the method for calculating the cumulative amplitude demonstrated its closer relationship with energetic electron fluxes as compared to the normal amplitude and attributed this to the presence of the cumulative effect of some diffusion processes: long-lived ULF wave activity is more important for electron acceleration than individual bursts of wave activity.

Note that the dependences illustrated in Figures 3, 4 are valid for the normal behavior of solar cycle. If it is abnormal, as it was during the anomalous minimum of 2008-2009, there were deviations from the regularities shown here.

\section{AN EXAMPLE OF SHORT-TERM FORECAST}

Let us give an example of the forecast that is based on the multivariate linear regression analysis of eight parameters acting as predictors. Unlike other studies relying on the multivariate statistical analysis [Simms et al., 2014, 2015, 2016; Sakaguchi et al., 2015], in this case, there is an additional element in the forecast procedure, which allows us to revise regression coefficients obtained from the preliminary analysis of regression relationships [Potapov et al., 2016].

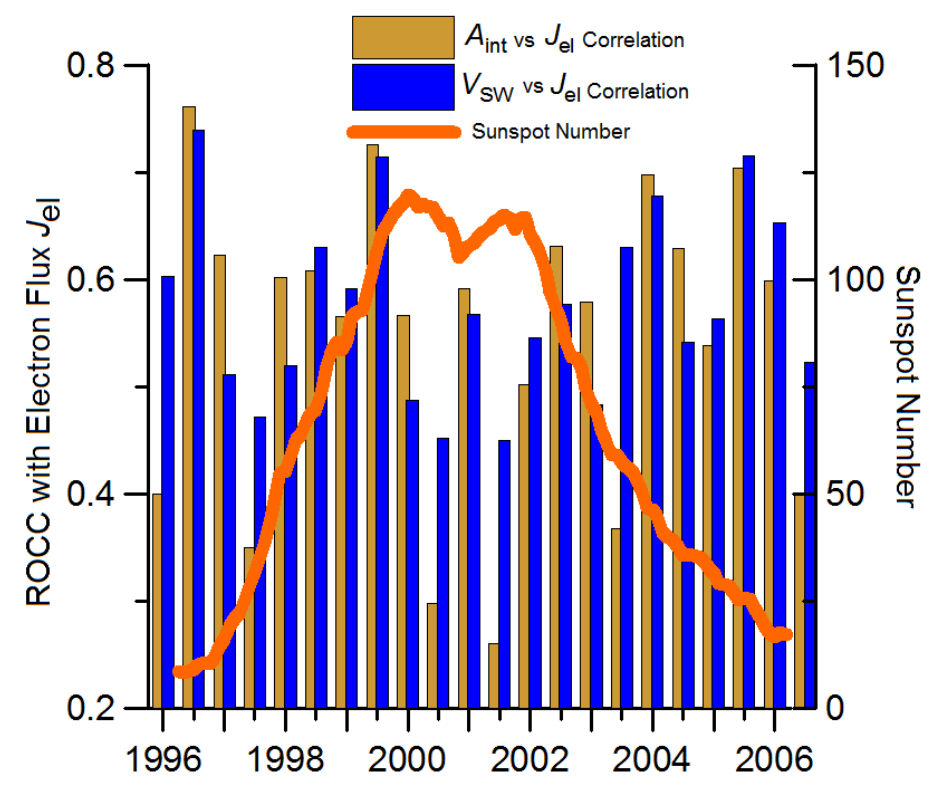

Figure 4. Semiannual values of the correlation coefficient between relativistic electron flux and solar wind velocity (blue bars) and with the cumulative on-ground amplitude of Pc5 ULF oscillations (brown bars) as compared to the variation in solar activity (orange curve) during solar cycle 23 [Potapov et al., 2014] 
As predictors we take daily averages of the following parameters: 1) solar wind velocity $\left(V_{\mathrm{sw}}\right)$; 2) amplitude of Pc45 ULF oscillations as inferred from ground-based $\left(A_{\mathrm{gr}}\right)$ and solar wind $\left(A_{\mathrm{sw}}\right)$ observations; 3$)$ horizontal component of the magnetic field at geosynchronous orbit $\left(H_{\mathrm{p}}\right)$; 4) density of solar wind protons $\left(N_{\mathrm{p}}\right)$; 5) solar wind dynamic pressure $\left.\left(p_{\text {dyn }}\right) ; 6\right)$ interplanetary electric field $\left.\left(E_{\mathrm{y}}\right) ; 7\right)$ maximum daytime flux $J_{600}$ of seed electrons with energy of about $600 \mathrm{keV}$, measured at geosynchronous orbit. The first four predictors have a forecast lead time $\Delta t$ equal to two days, for the last four, $\Delta t=1$ day. All raw data for the regression analysis and testing are taken over the period from 1996 to 2006. These data, after checking continuity of the time series, were split into two datasets. The former includes 184 daily measurements for the second half of 1999. This set is used to obtain initial coefficients for the multiple regression analysis. The latter entails 184 measurements for the second half of 2005. It also has no gaps in daily data. This set was used as a reference sample and to form an additional running window during the multiple regression analysis.

The conventional multiple regression analysis is as follows. A sample consisting of a number of independent parameters and one predictable parameter is employed to calculate coefficients for the multiple regression analysis. Resulting coefficients are utilized to compute values of the predictable parameter from the set of predictors from the reference sample. Thus, the reference sample simulates real-time measurements.

However, forecast results will be unsatisfactory, provided that the character of the relationship and, consequently, the regression coefficients between time intervals of data acquisition from master and reference samples change. To avoid this, in the described predictive model the regression coefficients are adjusted. For this purpose, an additional sample in the form of a 30-day running window is entered into the multiple regression analysis model. It is taken from the reference sample and is just before the forecast day, each time being shifted forward by one day. In the real forecast process, this running window follows the real day of forecast. Values of predictors of the additional sample are added to the master sample, and the joint sample is used to calculate new regression coefficients for each day. Figure 5 explains the scheme of the multiple regression analysis, through application of the running window [Potapov et al., 2016].

The model produces two types of forecast: two-day forecast based on the four predictors having a two-day lead time of the electron flux, and one-day forecast based on all the eight predictors.

Some results obtained by the predictive model are shown in Figure 6. The top panel (Figure 6, $a$ ) presents results of the estimated logarithm of the total daily relativistic electron flux $\lg J_{\mathrm{el}}$ from the model with the one-day forecast based on all the eight predictors. The solid black line indicates flux measurements, the dashed line depicts values derived from the regression coefficients calculated in the traditional multiple regression analysis, and the gray line shows estimations based on coefficients computed by the running window method. Figure $6, b, c$ illustrates the behavior of the squared deviation of forecast $\lg J_{\mathrm{f}}$ from actual flux values $\lg J_{\text {mes. }}$. As in Figure 6 , $a$, gray lines refer to forecasts made with the running window method; and dashed ones, with the traditional method. Two bottom panels also show values of the standard error of prediction $\sigma_{\text {est }}^{1}$ (standard forecast) and $\sigma_{\text {est }}^{2}$ (with running window). 


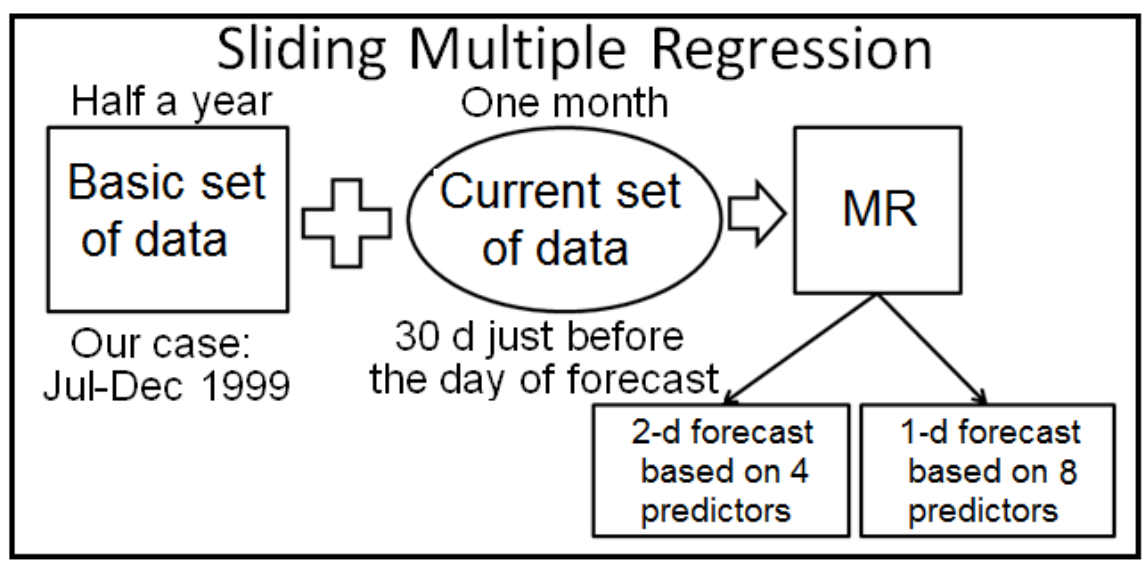

Figure 5. Flow diagram of sliding multiple regression [Potapov et al., 2016]

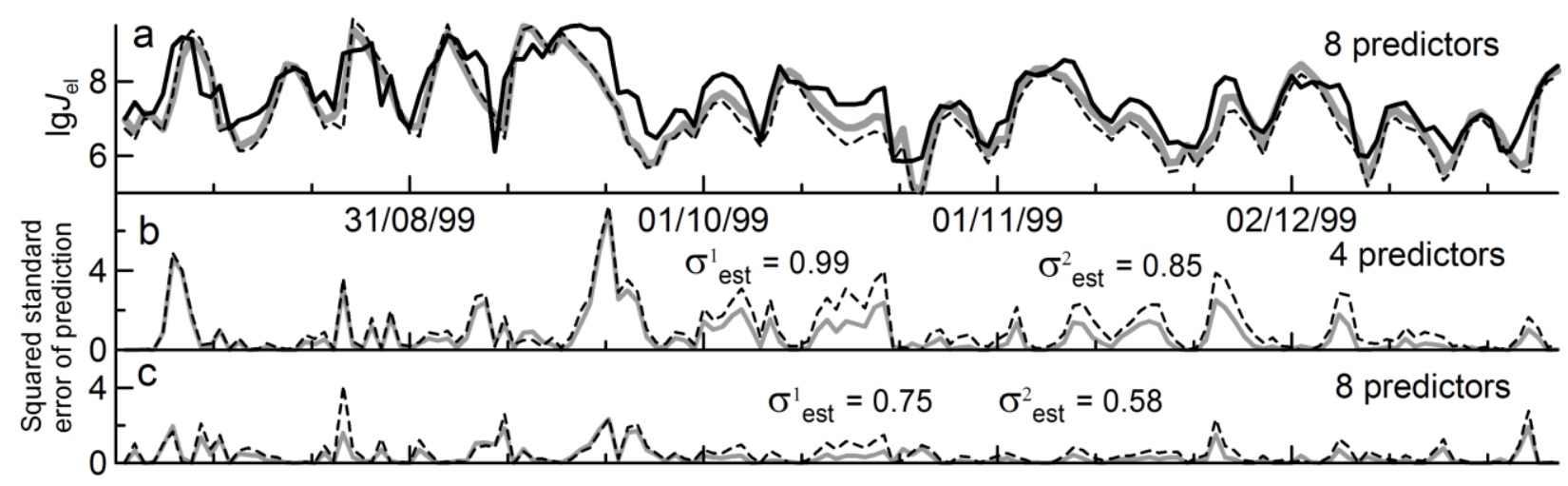

Figure 6. Results of the estimated logarithm of electron flux from the model with the one-day forecast based on eight predictors $(a)$; variations in squared deviation of forecast $\lg J_{\mathrm{f}}$ from actual flux values $\lg J_{\text {mes }}$ for the variant with the two-day forecast relying on four predictors $(b)$; the same for the variant with the one-day forecast based on eight predictors (c) [Potapov et al., 2016]

\section{CONCLUSION}

In conclusion, practical aspects of forecasting organization are discussed. Not all predictors are available in the real-time mode. For example, the geomagnetic index $A E$ is published on public websites not earlier than two weeks after recording, and only preliminary $K_{\mathrm{p}}$ and $D s t$ indices are accessible on-line. However, there is no tried and tested procedure for acquisition and distribution of data on some other predictors (say, the amplitude of the on-ground ULF or VLF oscillations). It is not surprising, therefore, that the only currently valid forecast is based on one predictor the solar wind velocity [http://www.swpc.noaa.gov/products/relativistic-electron-forecast-model], whereby its accuracy is low, and, as admitted by the authors of the method, errors in estimates especially increase during maximum solar activity when the magnetosphere is often attacked by high-speed solar wind streams.

Thus, organization of real-time forecasting capable of warning about possible threats to orbiting objects at geosynchronous orbit requires serious preparation. 
The first step is to choose the optimal forecast model using, where possible, on-line predictors, taking into account the lead time of forecast with them and their prognostic potential. Then, it is necessary to develop a reliable data acquisition system providing real-time data on parameters chosen as predictors. In elaborating the algorithm for prognostic estimates, it is desirable to make allowance for the regular correction of algorithm parameters (regression coefficients if the multiple regression method is employed) through application of running window or another method. Finally, we should regularly check the accuracy and reliability of the forecast, thus providing its real-time correction.

Medium-term forecasts impose other requirements. It is essential that they can, at least with $50 \%$ probability, predict the arrival of shock waves at Earth at the front of high-speed streams with two- or three-day lead time. This would give us a 3-5 day period of preparation for bursts of relativistic particles. Unfortunately, such reliability of medium-term forecast is unattainable.

In this review, we described the main characteristics of the electronic component of Earth's outer radiation belt in the geosynchronous orbit region, listed physical processes leading to electron acceleration, emphasized the need to develop high-energy electron forecasting methods in the said region, described the main potential predictors, and proposed an example of the predictive model for estimating electron fluxes with a 1-2-day lead time.

This work was supported by the Russian Foundation for Basic Research (grant No. 16-05-00631 and 16-05-00056) and the project No. 0344-2015-0018 of Program 7 of the RAS Presidium.

\section{REFERENCES}

Baker D.N. The occurrence of operational anomalies in spacecraft and their relationship to space weather. IEEE Trans. Plasma Sci. 2000, vol. 28, pp. 2007-2016.

Baker D. Satellite anomalies due to space storms. Space Storms and Space Weather Hazards / Ed. Daglis I.A. New York, Springer Publ., 2001, chap. 10, pp. 251-284.

Baker D.N., Higbie P.R., Belian R.D., Hones E.W. Do Jovian electrons influence the terrestrial outer radiation zone? Geophys. Res. Lett. 1979, vol. 6, pp. 531-534. DOI: 10.1029/ GL006i006p00531.

Baker D.N., Belian R.D., Higbie P.R., Klebesadel R.W., Blake J.B. Deep dielectric charging effects due to high energy electrons in the Earth's outer magnetosphere. J. Electrostatics. 1987, vol. 20, pp. 3-19.

Baker D.N., McPherron R.L., Cayton T.E., Klebesadel R.W. Linear prediction filter analysis of relativistic electron properties at 6.6 R $\mathrm{E}_{\mathrm{E}}$.J. Geophys. Res. 1990, vol. 95, no. A9, pp. 15133-15140. DOI: 10.1029/JA095iA09p15133.

Baker D.N., Allen J.H., Kanekal S.G., Reeves G.D. Disturbed space environment may have been related to Pager satellite failure. EOS Trans. AGU. 1998, p. 477.

Baker D.N., Kanekal S.G., Blake J.B., Pulkkinen T.I. The global efficiency of relativistic electron production in the Earth's magnetosphere. J. Geophys. Res. 2001, vol. 106, pp. 19169-19178.

Balikhin M.A., Boynton R.J., Walker S.N., Borovsky J.E., Billings S.A., Wei H.L. Using the NARMAX approach to model the evolution of energetic electrons fluxes at geostationary orbit. Geophys. Res. Lett. 2011, vol. 38, L18105. DOI: 10.1029/ 2011 GL048980.

Borovsky J.E., Cayton T.E., Denton M.H., Belian R.D., Christensen R.A., Ingraham J.C. The proton and electron radiation belts at geosynchronous orbit: Statistics and behavior during high-speed stream-driven storms. J. Geophys. Res.: Space Phys. 2016, vol. 121, pp. 5449-5488. DOI: 10.1002/ 2016JA022520. 
Brautigam D.H., Albert J.M. Radial diffusion analysis of outer radiation belt electrons during the October 9, 1990 magnetic storm. J. Geophys. Res. 2000, vol. 105, pp. 291-309. DOI: 10.1029/1999JA900344.

Degtyarev V.I., Chudnenko S.E., Kharchenko I.P., Tsegmed B., Xue B. Prediction of maximal daily average values of relativistic electron fluxes in geostationary orbit during the magnetic storm recovery phase. Geomagnetism and Aeronomy. 2009a, vol. 49, no. 8, pp. 1208-1217. DOI: 10.1134/ S0016793209080349.

Degtyarev V.I., Kharchenko I.P., Potapov A.S., Tsegmed B., Chudnenko S.E. Qualitative estimation of magnetic storm efficiency in producing relativistic electron flux in the Earth's outer radiation belt using geomagnetic pulsations data. Adv. Space Res. $2009 \mathrm{~b}$, vol. 43, pp. 829-836. DOI: 10.1016/j.asr. 2008.07.004.

Degtyarev V.I., Kharchenko I.P., Potapov A.S., Tsegmed B., Chudnenko S. E. The relation between geomagnetic pulsations and an increase in the fluxes of geosynchronous relativistic electrons during geomagnetic storms. Geomagnetism and Aeronomy. 2010, vol. 50, no. 7, pp. 885-893. DOI: 10.1134/ S0016793210070108.

Efitorov A., Myagkova I., Sentemova N., et al. Prediction of relativistic electrons flux in the outer radiation belt of the Earth using adaptive methods. Biologically Inspired Cognitive Architectures (BICA) for Young Scientists. Springer International Publ., 2016. P. 281-287. (Adv. Intelligent Systems and Computing. vol. 449). URL: http://link.springer.com/chapter/ 10.1007\%2F978-3-319-32554-5_36\#page-1 (accessed September 30, 2016).

Elkington S.R., Hudson M.K., Chan A.A. Acceleration of relativistic electrons via drift-resonant interaction with toroidalmode Pc-5 ULF oscillations. Geophys. Res. Lett. 1999, vol. 26, no. 21, pp. 3273-3276.

Fujimoto M., Nishida A. Energization and anisotropization of energetic electrons in the Earth's radiation belt by the recirculation process. J. Geophys. Res. 1990, vol. 95, no. A4, pp. 4265-4270. DOI: 10.1029/JA095iA04p04265.

Freeman J.W., O'Brien T.P., Chan A.A., Wolf R.A. Energetic electrons at geostationary orbit during the November 3-4, 1993 storm: Spatial/temporal morphology, characterization by a power law spectrum and, representation by an artificial neural network. J. Geophys. Res. 1998, vol. 103, pp. 26251-26260. DOI: 10.1029/97JA03268.

Friedel R.H.W., Reeves G.D., Obara T. Relativistic electron dynamics in the inner magnetosphere - A review. J. Atmos. Solar-Terr. Phys. 2002, vol. 64, pp. 265-282.

Gal'per A.M., Grachev V.M., Dmitrenko V.V., Kirillov-Ugryumov V.G., Ulin S.E. New component of the Earth's inner radiation belt: High-energy electrons. JETP Lett. 1983, vol. 38, no. 8, pp. 497-500.

Green J.C., Kivelson M.G. Relativistic electrons in the outer radiation belt: Differentiating between acceleration mechanisms. $J$. Geophys. Res. 2004, vol. 109, A03213. DOI: 10.1029/2003JA010153.

Gubar 'Yu.I. Drift resonance of relativistic electrons with ULF waves as a nonlinear resonance. Cosmic Res. 2010, vol. 48, no. 4, pp. 300-307. DOI: 10.1134/S0010952510040039.

Horne R.B., Thorne R.M. Potential waves for relativistic electron scattering and stochastic acceleration during magnetic storms. Geophys. Res. Lett. 1998, vol. 25, pp. 3011-3014. DOI: 10.1029/98GL01002.

Hudson M.K., Elkington S.R., Lyon J.G., Goodrich C.C. Increase in relativistic electron flux in the inner magnetosphere: ULF wave mode structure. Adv. Space Res. 2000, vol. 25, no. 12, pp. 2327-2337. DOI: 10.1016/S0273-1177(99)00518-9.

Kellerman A.C., Shprits Y.Y. On the influence of solar wind conditions on the outer-electron radiation belt. J. Geophys. Res. 2012, vol. 117, A05217. DOI: 10.1029/2011JA017253.

Kessel M. Things we do not yet understand about solar driving of the radiation belts. J. Geophys. Res.: Space Phys. 2016, vol. 121, pp. 5549-5552. DOI: 10.1002/2016JA022472.

Kuznetsov S.N. Izbrannye trudy po solnechno-zemnoi fizike [Selected Works on Solar-Terrestrial Phys.]. Moscow, Universitetskaya Kniga Publ., 2010, 256 p. (In Russian).

Li X., Temerin M., Baker D.N., Reeves G.D., Larson D. Quantitative prediction of radiation belt electrons at geostationary orbit based on solar wind measurements. Geophys. Res. Lett. 2001, vol. 28, pp. 1887-1890. DOI: 10.1029/2000GL012681.

Li L., Cao J., Zhou G. Combined acceleration of electrons by whistler-mode and compressional ULF turbulences near the geosynchronous orbit. J. Geophys. Res. 2005, vol. 110, A03203. DOI: 10.1029/2004JA010628.

Li L.Y., Cao J.B., Zhou G.C., Li X. Statistical roles of storms and substorms in changing the entire outer zone relativistic electron population. J. Geophys. Res. 2009, vol. 114, A12214. DOI: 10.1029/2009JA014333. 
Ling A.G., Ginet G.P., Hilmer R.V., Perry K.L. A neural network-based geosynchronous relativistic electron flux forecasting model. Space Weather. 2010, vol. 8, S09003. DOI: 10.1029/2010SW000576.

Lyatsky W., Khazanov G.V. Effect of geomagnetic disturbances and solar wind density on relativistic electrons at geostationary orbit. J. Geophys. Res. 2008, vol. 113, A08224. DOI: 10.1029/2008JA013048.

Lyons L.R., Lee D.-Y., Thorne R.M., Horne R.B., Smith A.J. Solar wind-magnetosphere coupling leading to relativistic electron energization during high-speed streams. J. Geophys. Res. 2005, vol. 110, A11202. DOI: 10.1029/2005JA011254.

Mann I.R., O’Brien T.P., Milling D.K. Correlations between ULF wave power, solar wind speed, and relativistic electron flux in the magnetosphere: Solar cycle dependence. J. Atmos. Solar-Terr. Phys. 2004, vol. 66, pp. 187-198.

Mathie R.A., Mann I.R. On the solar wind control of Pc5 ULF pulsation power at midlatitudes: Implications for MeV electron acceleration in the outer radiation belt. J. Geophys. Res. 2001, vol. 106, pp. 29783-29796.

Miyoshi Y., Kataoka R. Ring current ions and radiation belt electrons during geomagnetic storms driven by coronal mass ejections and corotating interaction regions. Geophys. Res.Lett. 2005, vol. 32, L21105. DOI: 10.1029/2005GL024590.

Miyoshi Y., Kataoka R. Flux enhancement of the outer radiation belt electrons after the arrival of stream interaction regions. $J$. Geophys. Res. 2008, vol. 113, A03S09. DOI: 10.1029/2007 JA012506.

Myagkova I.N., Dolenko S.A. Comparative analysis of the quality of prediction for fluences of relativistic electrons of the outer radiation belt of the Earth at different phases of the solar activity cycle. $11^{\text {th }}$ International Conference and School "Problems of Geocosmos": Book of Abstracts. St.-Petersburg, October 3-7, 2016. St.-Petersburg, 2016, p. 79.

Nagai T. Space weather forecast: Prediction of relativistic electron intensity at synchronous orbit. Geophys. Res. Lett. 1988, vol. 15 , pp. $425-428$.

O’Brien T.P., Lorentzen K.R., Mann I.R., Meredith N.P., Blake J.B., Fennell J.F., Looper M.D., Milling D.K., Anderson R.R. Energization of relativistic electrons in the presence of ULF power and MeV microbursts: Evidence for dual ULF and VLF acceleration. $J$. Geophys. Res. 2003, vol. 108, no. A8, pp. 2156-2202. DOI: 10.1029/2002JA009784.

Ozeke L.G., Mann I.R., Murphy K.R., Rae I.J., Milling D.K. Analytic expressions for ULF wave radiation belt radial diffusion coefficients. J. Geophys. Res.: Space Physics. 2014, vol. 119, pp. 1587-1605. DOI: 10.1002/2013JA019204.

Parker E.N. Geomagnetic fluctuations and the form of the outer zone of the Van Allen radiation belt. J. Geophys. Res. 1960, vol. 65, no. 10, pp. 3117-3130. DOI: 10.1029/ JZ065i010p03117.

Paulikas G.A., Blake J.B. Effects of the solar wind on magnetospheric dynamics: Energetic electrons at the synchronous orbit. Quantitative Modeling of Magnetospheric Processes. 1979. pp. 180-202. (Geophys. Monogr. Amer. Geophys. Un., Vol. 21).

Perry K.L., Ginet G.P., Ling A.G., Hilmer R.V. Comparing geosynchronous relativistic electron prediction models. Space Weather. 2010, vol. 8, S12002. DOI: 10.1029/2010SW000581.

Pilipenko V.A., Romanova N.V. The impact of space weather on the operation of spacecraft. Geofizicheskie issledovaniya [Geophys. Res.]. 2005, no. 2, pp. 71-82. (In Russian).

Pilipenko V., Yagova N., Romanova N., Allen J. Statistical relationships between the satellite anomalies at geostationary orbits and high-energy particles. Adv. Space Res. 2006, vol. 37, no. 6, pp. 1192-1205.

Potapov A.S. ULF wave activity in high-speed streams of the solar wind: Impact on the magnetosphere. J. Geophys. Res.: Space Phys. 2013, vol.118, no. 10, pp. 6465-6477. DOI: 10.1002/2013JA019119.

Potapov A.S., Tsegmed B., Ryzhakova L.V. Relationship between the fluxes of relativistic electrons at geosynchronous orbit and the level of ULF activity on the Earth's surface and in the solar wind during the 23rd solar activity cycle. Cosmic Res. 2012, vol. 50, no. 2, pp. 124-140. DOI: 10.1134/ S0010952512020086.

Potapov A.S., Tsegmed B., Ryzhakova L.V. Solar cycle variation of "killer" electrons at geosynchronous orbit and electron flux correlation with the solar wind parameters and ULF waves intensity. Acta Astronautica. 2014, vol. 93, pp. 55-63. DOI: 10.1016/j.actaastro.2013.07.004.

Potapov A.S., Ryzhakova L.V., Tsegmed B. A new approach to predict and estimate enhancements of "killer" electron flux at geosynchronous orbit. Acta Astronautica. 2016, vol. 126, pp. 47-51. DOI: 10.1016/j.actaastro.2016.04.017. 
Potapov A.S., Ryzhakova L.V., Tsegmed B. A method to forecast the relativistic electron flux at geostationary orbit. Vestnik SibGAU [Bull. of Siberian State Aerospace University]. 2016, vol. 17, no. 3, pp. 611-617. (In Russian).

Reeves G.D., McAdams K.L., Friedel R.H.W., O’Brien T.R. Acceleration and loss of relativistic electrons during geomagnetic storms. Geophys. Res. Lett. 2003, vol. 30, no. 10, 1529. DOI: 10.1029/2002GL016513.

Reeves G.D., Morley S.K., Friedel R.H.W., Henderson M.G., Cayton T.E., Cunningham G., Blake J.B., Christensen R.A., Thomsen D. On the relationship between relativistic electron flux and solar wind velocity: Paulikas and Blake revisited. J. Geophys. Res. 2011, vol. 116, A02213. DOI: 10.1029/ 2010JA015735.

Reeves G., Morley S., Cunningham G. Long-term variations in solar wind velocity and radiation belt electrons. J. Geophys. Res.: Space Physics 2013, vol. 118, no. 3, pp. 1040-1048. DOI: 10.1002/jgra.50126.

Roeder J.L., Fennell J.F., O'Brien T.P. Acceleration and losses of relativistic electrons due to whistler-mode chorus: SCATHA observations. AGU Fall Meeting. 2005, Abstract \# SM41D-07.

Romanova N., Pilipenko V. ULF wave indices to characterize the solar wind - magnetosphere interaction and relativistic electron dynamics. Acta Geophys. 2009, vol. 57, pp. 158-170. DOI: 10.2478/s11600-008-0064-4.

Romanova N.V., Pilipenko V.A., Yagova N.V., Belov A.V. Statistical correlation of the rate of failures on geosynchronous satellites with fluxes of energetic electrons and protons. Cosmic Res. 2005, vol. 43, no. 3, pp. 179-185. DOI: 10.1007/s10604-005-0032-6.

Romanova N., Pilipenko V., Crosby N., Khabarova O. ULF wave index and its possible applications in space physics. Bulg. J. Phys. 2007, vol. 34, pp. 136-148.

Romanova N.V., Chizhenkov V.A., Pilipenko V.A. Possible relation of emergencies during spacecraft launches from the Plesetsk site to high-latitude geomagnetic disturbances. Geomagn. Aeron. 2009, vol. 49, no. 1, pp. 104-109. DOI: 10.1134/S0016793209010149.

Sakaguchi K., Nagatsuma T., Reeves G.D., Spence H.E. Prediction of MeV electron fluxes throughout the outer radiation belt using multivariate autoregressive models. Space Weather. 2015, vol. 13, pp. 853-867. DOI: 10.1002/ 2015SW001254.

Schulz M., Lanzerotti L. Particle Diffusion In The Radiation Belts. Berlin, Springer Pabl., 1974, 218 p.

Sheldon R.B., Spence H.E., Sullivan J.D., Fritz T.A., Chen J. The discovery of trapped energetic electrons in the outer cusp. $G e$ ophys. Res. Lett. 1998, vol. 25, no. 11, pp. 1825-1828.

Shiroky V.R., Dolenko S.A., Myagkova I.N., Sentemova N.S. A study of neural network forecasting horizon of the Earth's magnetosphere state, XVIII International Scientific and Technical Conference "Neuroinformatika-2016", Collection of scientific papers. In 3 parts. Moscow, NIYU MIPhI Pabl., 2016, Pt. 1, pp. 172-182. (In Russian).

Shprits Y., Drozdov A.Y., Spasojevic M., Kellerman A.C., Usanova M.E., Engebretson M.J., Agapitov O.V., Orlova K.G., Zhelavskaya I.S., Raita T., Spence H.E., Baker D.N., Zhu H. Wave-induced loss of ultra-relativistic electrons in the Van Allen radiation belts. Nature Communications. 2016, vol. 7, 12883. DOI: 10.1038/ ncomms12883.

Simms L.E., Pilipenko V., Engebretson M.J., Reeves G.D., Smith A.J., Clilverd M. Prediction of relativistic electron flux at geostationary orbit following storms: Multiple regression analysis. J. Geophys. Res.: Space Phys. 2014, vol. 119, no. 9, pp. 7297-7318. DOI: 10.1002/2014JA019955.

Simms L.E., Engebretson M.J., Smith A.J., Clilverd M., Pilipenko V., Reeves G.D. Analysis of the effectiveness of groundbased VLF wave observations for predicting or nowcasting relativistic electron flux at geostationary orbit. J. Geophys. Res.: Space Phys. 2015, vol. 120, pp. 2052-2060. DOI: 10.1002/ 2014JA020337.

Simms L.E., Engebretson M.J., Pilipenko V., Reeves G.D., Clilverd M. Empirical predictive models of daily relativistic electron flux at geostationary orbit: Multiple regression analysis. J. Geophys. Res.: Space Phys. 2016, vol. 121, pp. 3181-3197. DOI: 10.1002/2016JA022414.

Summers D., Ma C. A model for generating relativistic electrons in the Earth's inner magnetosphere based on gyroresonant waveparticle interactions. J. Geophys. Res. 2000, vol. 105, no. A2, pp. 2625-2640. DOI: 10.1029/ 1999JA900444.

Summers D., Ni B., Meredith N.P. Timescales for radiation belt electron acceleration and loss due to resonant waveparticle interactions: 2. Evaluation for VLF chorus, ELF hiss, and electromagnetic ion cyclotron waves. J. Geophys. Res. 2007, vol. 112, A04207. DOI: 10.1029/2006JA011993. 
Temny V.V. History of the discovery of the Earth's radiation belts: Who, when and how? Zemlya $i$ Vselennaya [Earth and Universe]. 1993, no. 5, pp. 69-76. (In Russian).

Turner D.L., Morley S.K., Miyoshi Y., et al. Outer radiation belt flux dropouts: Current understanding and unresolved questions. Dynamics of Earth's Radiation Belts and Inner Magnetosphere. Ed. by D. Summers et al. 2012, pp. 195-212. (Geophys. Monogr. Ser., Vol. 199). DOI: 10.1029/2012GM001310.

Tverskoy B.A. Capture of fast particles from interplanetary space. Izv. AN SSSR. Ser. fiz. [Bull. Russian Academy of Sciences. Physics]. 1964, vol. 28, pp. 2099-2103. (In Russian).

Tverskoy B.A. Dinamika radiatsionnykh poyasov Zemli [Dynamics of the Earth's radiation belts]. Moscow, Nauka Publ., 1968. 224 p. (In Russian).

Ukhorskiy A.Y., Sitnov M.I., Sharma A.S., Anderson B.J., Ohtani S., Lui A.T.Y. Data-derived forecasting model for relativistic electron intensity at geosynchronous orbit. Geophys. Res. Lett. 2004, vol. 31, L09806. DOI: $10.1029 / 2004$ GL019616.

Ukhorskiy A.Y., Sitnov M.I., Millan R.M., Kress B.T., Smith D.C. Enhanced radial transport and energization of radiation belt electrons due to drift orbit bifurcations. J. Geophys. Res.: Space Physics. 2014. vol. 119, pp. 163-170. DOI: 10.1002/ 2013JA019315.

Ukhorskiy A. Y., Sitnov M.I., Millan R.M., Kress B.T., Fennell J.F., Claudepierre S.G., Barnes R.J. Global storm time depletion of the outer electron belt. J. Geophys. Res.: Space Phys. 2015, vol. 120, pp. 2543-2556. DOI: 10.1002/2014JA020645.

Weigel R.S., Klimas A.J., Vassiliadis D. Precursor analysis and prediction of large-amplitude relativistic electron fluxes. Space Weather. 2003, vol. 1, p. 1014. DOI: 10.1029/2003SW000023.

Xiao F., Shen C., Wang Y., Zheng H., Wang S. Energetic electron distributions fitted with a relativistic kappa-type function at geosynchronous orbit. J. Geophys. Res. 2008, vol. 113, A05203. DOI: 10.1029/2007JA012903.

URL: https://encrypted-tbn0.gstatic.com/images?q=tbn:ANd9GcQRReeeIY4NuZm-hTm8qUT6aNUkZ53jtpe Cd8eo6dpL88X2DVNZw/ (accessed September 30, 2016).

URL: http://www.swpc.noaa.gov/products/relativistic-elect-ron-forecast-model/ (accessed September 30, 2016).

URL: http://ulf.gcras.ru/ (accessed September 30, 2016). 